\title{
MGSGenerator 1.5: software tool for reconstructing mathematical models of metabolic networks
}

\author{
F.V. Kazantsev \\ Kurchatov genomics center \\ ICG SB RAS \\ Novosibirsk, Russia \\ kazfdr@bionet.nsc.ru
}

\author{
S.A. Lashin \\ Kurchatov genomics center \\ ICG SB RAS \\ Novosibirsk, Russia, \\ Novosibirsk State University \\ Novosibirsk, Russia \\ lashin@bionet.nsc.ru
}

\begin{abstract}
There are lots of sources of information on metabolic networks but not so many sources dealing with existing mathematical models in spite of the fact that the modeling approach is an essential way for metabolic networks analysis. One of the ways to get ready-to-simulate frame models is the using of template approaches for mathematical model reconstruction, which take into account the nature of metabolic processes. However, building of more precise models typically requires reusing of existing models already adapted to experimental data. The aim of our research is to develop a tool that makes complete ready-to-simulate mathematical model for an input metabolic network. We present MGSGenerator 1.5 as the tool relied on API of existing model sources.
\end{abstract}

Keywords - Mathematical model, E.coli, API, model generation, metabolic networks

\section{Motivation and Aim}

Mathematical modeling is widely used in microbiology investigations for metabolic networks analysis for the substance yield forecasting or adjustment appropriate environment conditions. In silico simulation allows us to check hundreds parameters for a model in a short period of time, depending on available computational environments.

Metabolic network reconstruction/analysis is the part of genome annotation steps in microbiology research. In this work we focus on the steps that go after. Metabolic network is the structural (graph) model that shows which substances interact. We are interested in the analysis of those interactions in time and in space. For the purpose, we should process structural model into dynamic model, which may be formalized in different forms. In this study we obtain Ordinary Differential Equation (ODE) model. One of the ways to get ready-tosimulate frame ODE models is the using of template approaches based on the standard equations for various types of metabolic processes. However, building of more precise ones typically requires reusing of existing models already adapted to experimental data.

The aim of our research is to develop a tool that makes complete ready-to-simulate mathematical model for an input metabolic network. The tool should be able to address API of existing model sources.

\section{Results}

We have advanced our previous tool MGSGenerator [1] to generate frame models. The novel version is 1.5 . The tool processes the following steps:

- It takes structural model of metabolic network as an input. It decomposes network on subsystems and processes each of them.

- $\quad$ Each subsystem is compared with the set of existing models taken from various sources. The main source at present is MAMMOTh database [2] containing mathematical models of enzymatic reaction of $E$. coli adapted to experimental data.

- If appropriate structure model was not found, then the rule-based frame mathematical model generation process starts.

- To provide ready-to-simulate model, processes of substances sink and inflow are added.

- Finally, we obtain SBML model that then could be explored by Copasi tool (copasi.org) or any other simulation tools supporting SBML import.

We have tested the tool with metabolic networks of different size, from single metabolic pathway to the wholegenome metabolic network.

\section{ACKNOWLEDGMENT}

The study was supported by the Budget Project No. 03242019-0040-C-01 and Kurchatov Genomics Center of ICG SB RAS.

\section{REFERENCES}

[1] F. Kazantsev, I. Akberdin, K. Bezmaternykh, V. Likhoshvai, (2009) "The tool for automatic generation of gene networks mathematical models", VOGiS Herald, 13(1):163-169 [in Russian]

[2] F. Kazantsev, I. Akberdin, S. Lashin, N. Ree, V. Timonov, A Ratushnyi, T. Khlebodarova, and V. Likhoshvai, (2018) "MAMMOTh: a new database for curated mathematical models of biomolecular systems", Journal of Bioinformatics and Computational Biology, 16(01): 1740010, https://doi.org/10.1142/S02197200174001 